6 Vilkman S, Keistinen T, Tuuponen T, et al. Survival and cause of death among elderly chronic obstructive disease patients after first admission to hospital. Respiration 1997;64:281-4.

7 Zielinski J, MacNee W, Wedzicha J, et al. Causes of death in patients with COPD and chronic respiratory failure. Monaldi Arch Chest Dis 1997;52:43-7.

8 Hansell AL. Lies, damned lies and mortality statistics? Thorax 2006;61:923-4.

9 Sin DD, Man SFP. Cooling the fire within: inhaled corticosteroids and cardiovascular mortality in COPD. Chest 2006;130:629-30.

10 Macie C, Wooldrage K, Manfreda J, et al. Inhaled corticosteroids and mortality in COPD. Chest 2006; 130:640-6.

11 Price LC, Lowe D, Hosker HSR, et al, on behalf of the British Thoracic Society and the Royal College of Physicians Clinical Effectiveness Evaluation Unit (CEEu). UK National COPD Audit 2003: impact of hospital resources and organisation of care on patient outcome following admission for acute COPD exacerbation. Thorax 2006:61:837-42.

12 Mannino DM. Women and chronic obstructive pulmonary disease: does sex influence survival? Am J Respir Crit Care Med 2006;174:488-9.

13 Machado ML, Krishman JA, Buist AS, et al. Sex differences in survival in oxygen-dependent patients with chronic obstructive pulmonary disease. Am J Respir Crit Care Med 2006; 174:524-9.

14 Ramsey SD, Hobbs FDR. Chronic obstructive pulmonary disease, risk factors, and outcome trials: comparisons with cardiovascular disease. Proc Am Thorac Soc 2006:3:635-40.

15 McGarvey LP, John M, Anderson JA, et al. Ascertainment of cause-specific mortality in COPD: operations of the TORCH Clinical Endpoint Committee. Thorax 2007;62:411-15.
16 Sin DD, Wu L, Man SFP. The relationship between reduced lung function and cardiovascular mortality: a population-based study and a systematic review of the literature. Chest 2005; 127:1952-9.

17 Hole DJ, Watt GC, Davey-Smith G, et al. Impaired lung function and mortality risk in men and women findings from the Renfrew and Paisley prospective population study. BMJ 1996;313:711-5.

18 Curkendall SM, Deluise C, Jones JK, et al. Cardiovascular disease in patients with chronic obstructive pulmonary disease, Saskatchewan Canada Cardiovascular Disease in COPD patients. Ann Epidemiol 2006; 16:63-70.

19 Soyseth V, Brekke PH, Smith P, et al. Statin use is associated with reduced mortality in COPD. Eur Respir J 2007;29:279-83.

20 Laver MS, Blackstone EH, Young JB, et al. Cause of death in clinical research: time for a reassessment? J Am Coll Cardiol 1999;34:618-20.

\section{Abbreviated or not abbreviated? Is it the right question?}

\section{Frederic Sériès}

\section{The use of abbreviated recording techniques in the diagnosis of sleep-disordered breathing}

$\mathrm{S}$ leep-disordered breathing (SDB) disturbances are very prevalent in developed countries. Since it was estimated over 10 years ago, the prevalence of SDB is probably higher now because of the dramatic increase in body weight in the populations of these countries. ${ }^{1}$ Given the large increase in mortality and morbidity outcomes associated with the diagnosis of SDB, the diagnosis of a nocturnal breathing disorder should no longer be confirmed solely by conventional in-laboratory polysomnographic recordings. This justifies the need for abbreviated monitoring during sleep to be part of the assessment of SDB and the tremendous effort developed by the sleep research community to evaluate the diagnostic value of abbreviated recordings.

The study by Jobin et $a^{2}$ reported in this issue of Thorax (see $p$ 422) is the first comparative study that does not use inlaboratory polysomnographic recordings as the gold standard, and is thus an important step towards evaluating the merits of abbreviated recording techniques. This is a major upheaval in the field of sleep medicine, and opens the way to realistic assessments of abbreviated recording techniques in real-life conditions that avoid costly, time-consuming inlaboratory polysomnographic recordings.
It is, however, reasonable to wonder whether the authors should have proceeded more cautiously by starting with level 2 monitoring techniques (ie, an unattended complete polysomnographic study) as a reference, which would allow the influence of home monitoring on cardiorespiratory variables to be evaluated while, at the same time, taking potential differences in sleep characteristics into consideration. The authors did not explain why electrophysiological variables, which can be recorded using the Suzanne apparatus, were not collected. At a minimum, the reference portable monitoring technique should be designed to interfere minimally with sleep quality. The level 3 device used by Jobin et al may not fully meet these requirements due to the cumbersome equipment, but the latest generation of recording systems should correct these potential pitfalls

Despite the tremendous interest in the use of abbreviated monitoring by the medical community, American medical societies (APSS, ACCP, ATS) have, until recently, maintained that portable monitoring devices are not accurate enough to be used in an ambulatory setting for the management of SDB. ${ }^{3}$ A number of reasons may account for the discrepancy between the official recommendations of medical societies and the widespread use of abbreviated monitoring by the medical sleep community (apart from the potential impact of differences in reimbursement rules in certain countries). One is the very large disparity in the recorded signals and in the recording and signal processing techniques of the devices that have been tested (such as oximetry, breathing sounds, sophisticated cardiac rhythm analysis (heart rate variability), respiratory impedance signals, pulse transit time, arterial tonometry). In this regard, night-time oximetry recordings remain the most extensively investigated technique, and it is somewhat paradoxical that a typical desaturation/resaturation profile per se may not be considered as a diagnostic finding given that a repetitive fall in arterial oxygen saturation $\left(\mathrm{SaO}_{2}\right)$ is recognised as a cornerstone of the capacity of sleep recordings to identify $\mathrm{SDB}^{4}$ and that the accuracy of $\mathrm{SaO}_{2}$ recording techniques (probes, software analysis including artefact deletion, sampling frequency, averaging time, signal processing) has dramatically improved in recent years.

The discrepancies in the diagnostic performance of oximetry recording techniques reflect the specificity of the data obtained with a given recording system, but also indicate the need to have access to, and to examine, raw data to satisfactorily interpret abbreviated recordings. Considering that "oximetry" refers to a wide variety of different techniques with different diagnostic performances, ${ }^{5}$ the term "oximetry" is meaningless when used to designate an investigation category. The work of Jobin et al illustrates this point since the desaturation profiles of the two oximeters they tested were different. Expertise with portable monitoring thus has to be developed in each sleep centre and should take into account the usefulness and limits of portable monitoring devices in the investigation strategy for individual patients. 
Another concern is the moving target of the validation of technological development procedures, particularly with respect to the choice of the signal(s) to be recorded and the way tracings should be scored. One example of this is the lack of change in the normal threshold of the apnoea-hypopnoea index when highly sensitive signals such as nasal pressure recordings are used instead of the less sensitive signals used to select the 5 events/h threshold. ${ }^{4}$

A further reason for the discrepancy between official recommendations and the widespread use of portable monitoring is the unrealistic diagnostic value that has been attributed to abbreviated recording techniques in the investigation of SDB; that is, that the results of these recordings have usually been considered in the binary fashion where a negative test is useless in the diagnosis of sleep apnoea. However, it must be pointed out that portable monitors have been extensively tested in patients who are referred to tertiary centres independent of underlying symptoms and morbid conditions. This is not the ideal situation for using these devices. The circumstances of individual patients (logistical considerations, ability to install captors and to check whether the signals are adequate) should be taken into consideration, as should the factors known to influence the variables being measured. For example, in the presence of SDB, the potential of abnormal nocturnal oximetry is probably negatively influenced by the absence of excess weight and/or comorbid conditions. The same reasoning applies to the investigation of patients whose comorbid conditions may be worsened by SDB, independent of any vigilance disturbances. This may be a growing problem since cardiovascular and metabolic morbidity factors have been linked to the number of nocturnal desaturation/resaturation events ${ }^{4}$ through their effect on systemic inflammation and sympathetic activity. ${ }^{78}$ In situations where diurnal somnolence is not an issue, oximetry may be the test of choice because, unlike sleepiness investigation strategies, negative oximetry recordings eliminate the contribution of nocturnal breathing disorders as a deleterious factor of a patient's comorbidity. Diagnostic studies conducted in these populations, rather than in patients referred to sleep clinics, should help to refine these diagnostic criteria by targeting patients who best fit the diagnostic value of a given abbreviated recording technique. Combined with an increase in the availability of portable monitors, this would appear to be the only effective strategy to reduce investigation delays and costs. This further implies that training and educational strategies adapted to the condition of individual patients are required for poly-

somnography to no longer be the "one size fits all" approach to investigating SDB.

Abbreviated recording techniques should become an integral part of a stepwise investigation strategy where the results of sleep recordings must respond to the questions raised by the clinician about a patient's specific clinical condition and pre-test probability. Future approaches should thus take into account the condition of individual patients and the clinical justification for conducting ambulatory recordings during sleep to select the most appropriate investigation strategy and to refine the diagnostic procedure by using level 4 to level 1 recording techniques in a stepwise fashion.

Thorax 2007;62:379-380.

doi: $10.1136 /$ thx.2006.064808

Correspondence to: Dr Frederic Sériès, Centre de Pneumologie, Hôpital Laval, 2725 Chemin Sainte-Foy, Sainte-Foy, Quebec, Canada GIV 4G5; frederic.series@med.ulaval.ca

Competing interests: None.

Dr Sériès is a scholar of the Fonds de Recherche en Santé du Québec.

\section{REFERENCES}

1 Hiestand DM, Britz P, Goldman M, et al. Prevalence of symptoms and risk of sleep apnea in the US population: results from the National Sleep Foundation Sleep in America 2005 poll. Chest 2006;130:780-6.

2 Jobin V, Mayer P, Bellemare F. Predictive value of automated oxygen saturation analysis for the diagnosis and treatment of obstructive sleep apnoea in a home-based setting. Thorax 2007:62:422-7.

3 Anon. Executive summary on the systematic review and practice parameters for portable monitoring in the investigation of suspected sleep apnea in adults. Am J Respir Crit Care Med 2004;169:1 160-3.

4 Newman AB, Nieto FJ, Guidry U, et al. Relation of sleep-disordered breathing to cardiovascular disease risk factors: the Sleep Heart Health Study. Am J Epidemiol 2001;154:50-9.

5 Zafar S, Ayappa I, Norman RG, et al. Choice of oximeter affects apnea-hypopnea index. Chest $2005 ; 127: 80-8$.

6 Resnick HE, Redline S, Shahar E, et al. Diabetes and sleep disturbances: findings from the Sleep Heart Health Study. Diabetes Care 2003;26:702-9.

7 Ryan S, Taylor CT, MCNicholas WT. Predictors of elevated nuclear factor-kappaB-dependent genes in obstructive sleep apnea syndrome. Am J Respir Crit Care Med 2006; 174:824-30.

8 Levenberger UA, Brubaker D, Quraishi S, et al. Effects of intermittent hypoxia on sympathetic activity and blood pressure in humans. Auton Neurosci 2005;121:87-93.

\section{Pulmonary puzzles}

\section{Mark Fitzgerald, Wisia Wedzicha}

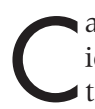
ase reports are a useful method of identifying unique case presentations, often with important clinical caveats. Unfortunately, with the pressure on space, especially in high impact journals such as Thorax, the ability to accept case reports for publication is extremely low. A number of years ago Thorax, recognising its inability to publish more than a minority of case reports submitted, introduced what has transpired to be the very successful "Images in Thorax" section. Despite this, the pressure to publish case reports continues, unabated.

Recognising that there will continue to be a need for innovative case reports, especially those that speculate on innovative new hypotheses, we feel that there is a role for a more educational format for case reports. Therefore, unless a case report has unique content and, in particular, provides data not previously reported, it should be changed into a "Pulmonary puzzles". These puzzles will consist of a brief clinical summary and appropriate image, from which the reader will be asked to provide a possible diagnosis. The reader will then be referred to another page later in the same issue of Thorax where the key diagnostic test(s) will be provided along with a brief 